\title{
Body composition assessment: comparison of quantitative values between magnetic resonance imaging and computed tomography
}

\author{
Chiara Zaffina ${ }^{1}$, Rolf Wyttenbach ${ }^{2,3,4}$, Alberto Pagnamenta ${ }^{5,6,7}$, Rosario Francesco Grasso ${ }^{8}$, Matteo Biroli ${ }^{9}$, \\ Filippo Del Grande ${ }^{2,3}$, Stefania Rizzo ${ }^{2,3} \wedge$ \\ ${ }^{1}$ Policlinico Universitario Campus Bio-Medico di Roma, Roma, Italy; ${ }^{2}$ Istituto di Imaging della Svizzera Italiana (IIMSI), Ente Ospedaliero \\ Cantonale, Lugano, Switzerland; ${ }^{3}$ Facoltà di Scienze Biomediche, Università della Svizzera Italiana, Lugano, Switzerland; ${ }^{4}$ Department of \\ Diagnostic, Interventional and Pediatric Radiology (DIPR), Inselspital, Bern University Hospital, University of Bern, Bern, Switzerland; ${ }^{5}$ Clinical \\ Trial Unit, Ente Ospedaliero Cantonale, Lugano, Switzerland; ${ }^{6}$ Intensive Care Unit, Ente Ospedaliero Cantonale, Mendrisio, Switzerland; ${ }^{7}$ Division \\ of Pneumology, University of Geneva, Geneva, Switzerland; ${ }^{8}$ Departmental Faculty of Medicine and Surgery, Unit of Interventional Radiology, \\ Università Campus Bio-Medico di Roma, Rome, Italy; ${ }^{9}$ Humanitas University, Department of Biomedical Sciences, Pieve Emanuele, Milan, Italy
}

Contributions: (I) Conception and design: S Rizzo, C Zaffina, F Del Grande, R Wyttenbach; (II) Administrative support: F Del Grande; (III) Provision of study materials or patients: S Rizzo, C Zaffina, M Biroli, A Pagnamenta; (IV) Collection and assembly of data: S Rizzo, C Zaffina, M Biroli; (V) Data analysis and interpretation: S Rizzo, C Zaffina, A Pagnamenta, R Wyttenbach; (VI) Manuscript writing: All authors; (VII) Final approval of manuscript: All authors.

Correspondence to: Stefania Rizzo, PD, Dr. Med. Clinica di Radiologia EOC, Istituto di Imaging della Svizzera Italiana (IIMSI), Ente Ospedaliero Cantonale, Via Tesserete 46, Lugano 6900, Switzerland. Email: stefania.rizzo@eoc.ch.

Background: The primary objective of this study was to compare measurements of skeletal muscle index (SMI), visceral adipose tissue (VAT) and subcutaneous adipose tissue (SAT) at the level of L3, on subjects who underwent computed tomography (CT) and magnetic resonance imaging (MRI) examinations within a three-month period. The secondary objective was to compare the automatic and semi-automatic quantifications of the same values for CT images.

Methods: Among subjects who underwent CT and MRI at our Institution between 2011 and 2020, exclusion criteria were: presence of extensive artifacts; images not including the whole waist circumference; CT acquired with low-dose technique and lack of non-contrast images. A set of three axial images (CT, MRI T1-weighted and T2-weighted) were used to extract the following measurements with semi-automatic segmentations: SMI [calculated normalizing skeletal muscle area (SMA) by the square height], SAT, VAT. For the CT images only, the same values were also calculated by using automatic segmentation. Statistical analysis was performed comparing quantitative MRI and CT measurements by Pearson correlation analysis and by Bland-Altman agreement analysis.

Results: A total of 123 patients were included. By performing linear regression analysis, CT and MRI measurements of SMI showed a high correlation $\left(r^{2}=0.81\right.$ for $T 1, r^{2}=0.89$ for $\left.T 2\right)$, with a mean logarithmic difference between CT and MRI quantitative values of 0.041 for T1-weighted and 0.072 for T2-weighted images. CT and MRI measurements of SAT showed high correlation $\left(r^{2}=0.81\right.$ for $T 1 ; r^{2}=0.81$ for T2), with a mean logarithmic difference between CT and MRI values of 0.0174 for T1-weighted and 0.201 for T2weighted images. CT and MRI measurements of VAT showed high correlation $\left(r^{2}=0.94\right.$ for T1; $r^{2}=0.93$ for T2), with a mean logarithmic difference of 0.040 for T1-weighted and -0.084 for T2-weighted images. The comparison of values extracted by semi-automatic and automatic segmentations were highly correlated.

Conclusions: Quantification of body composition values at MRI from T1-weighted and T2-weighted images was highly correlated to same values at CT, therefore quantitative values of body composition among

\footnotetext{
$\wedge$ ORCID: 0000-0002-5151-0866.
} 
patients who underwent either one of the examinations may be compared. CT body composition values extracted by semi-automatic and automatic segmentations showed high correlation.

Keywords: Body composition; computed tomography (CT); magnetic resonance imaging (MRI)

Submitted Jun 13, 2021. Accepted for publication Nov 11, 2021.

doi: 10.21037/qims-21-619

View this article at: https://dx.doi.org/10.21037/qims-21-619

\section{Introduction}

Body composition may be an important feature in many clinical settings as it can be associated with efficacy and toxicity of therapies, with patient functional status as well as with surgical complication rates and survival (1-3). Many studies have calculated body composition in different ways, ranging from a simple waist-to-hip ratio or body mass index (BMI) to a complex profiling, usually reserved for research settings, based on fat-referenced magnetic resonance imaging (MRI) (4).

Since water, fat and proteins compose the soft tissues of the human body, body composition measurements include segmentation of muscle, visceral fat and subcutaneous fat, frequently performed on a single axial computed tomography (CT) particularly in oncology research (5-9). Since the muscles at the level of L3 include the psoas muscle, the paraspinal muscles and the abdominal wall muscles, the skeletal muscle area (SMA) at this level is considered an optimal approximate for the entire body skeletal muscle (5), and allows to calculate skeletal muscle index (SMI), as SMA divided by square height. The distinction of subcutaneous adipose tissue (SAT) and visceral adipose tissue (VAT) is important because the two types of fat accumulation are associated with different metabolic risks (10-12).

All these measurements can be performed by using semiautomatic or fully automatic segmentation methods on CT. Since CT imaging is based on the use of X-rays, its use may be a hurdle to clinical research in healthy volunteers, as well as in patients that are evaluated solely by MRI (13).

Compared to CT, MRI shows better soft tissue definition, especially for fat, and thus it may offer easier segmentation of fat and muscles $(14,15)$, although its use for body composition assessment is still limited because of the costs involved and the need for more complex postprocessing techniques (4).

However, in clinical and research settings where either CT or MRI are available, it would be of benefit to know whether body composition measures evaluated on routine clinical abdominal MRI and CT at the level of the third lumbar vertebra (L3), may be as reliable.

Therefore, the primary objective of this study was to retrospectively compare measurements of SMI, VAT and SAT at the level of L3, on subjects who underwent clinical CT and MRI examinations within a 3 -month period. The secondary objective was to compare the automatic and semi-automatic quantifications of the same values for the sole CT images.

We present the following article in accordance with the MDAR checklist (available at https://dx.doi.org/10.21037/ qims-21-619).

\section{Methods}

\section{Patient selection}

The study was conducted in accordance with the Declaration of Helsinki (as revised in 2013). The study was approved by the regional ethics committee of Canton Ticino and individual consent for this retrospective analysis was waived. The study population was retrospectively selected among subjects who underwent both a CT and an MRI scan for medical purposes at our Institution within no more than 3 months between them between 2020 and 2011 . We did not go furtherly backwards because the techniques of acquisition could have been different, thus influencing the quality of segmentation (16). The mean, median and standard deviation (SD) of time interval between the two exams were calculated and evaluated for significance. The height of the patients was recorded. The weight of the patients was recorded, where available, at the two timepoints and mean, median and SD were calculated. Clinical indication to perform the examination was also recorded. Exclusion criteria were: presence of extensive artifacts due to metallic prostheses (for CT and MRI) or bowel movements (for MRI); images not including the whole waist circumference at the level of L3 (for CT and MRI); CT 
Table 1 Sarcopenia defined according to BMI and SMI threshold values (Martin)

\begin{tabular}{lcc}
\hline \multirow{2}{*}{ BMI } & \multicolumn{2}{c}{ SMI index } \\
\cline { 2 - 3 } & Male & Female \\
\hline$<20.0$ & $<43$ & $<41$ \\
$20.0-24.9$ & $<43$ & $<41$ \\
$25.0-29.9$ & $<53$ & $<41$ \\
$>29.9$ & $<53$ & $<41$ \\
\hline
\end{tabular}

BMI, body mass index; SMI, skeletal muscle index.

acquired with low-dose technique; lack of sequence without contrast medium at CT.

Body surface area (BSA) was calculated according to the Du Bois formula (17). BMI was calculated as weight/ height ${ }^{2}$. Patients were then further divided into three groups for separate analyses according to $\mathrm{WHO}$ definition of obesity (18) into: underweight (BMI: <18.5), normal weight (BMI: 18.5-24.9), overweight or obese (BMI: >25). A further distinction was made between sarcopenic and non sarcopenic patients according to the criteria derived from Martin and colleagues (19), as shown in Table 1.

\section{CT and MRI acquisition}

CT and MRI examinations were acquired on different scanners at different Institutions, but they were all available in digital format on the local picture archiving and computer system (PACS). The CT series used for extraction were acquired without iodinated contrast medium; the MRI sequences used were T1-weighted and T2-weighted without fat saturation, acquired on $3 \mathrm{~T}$ machines.

\section{CT and MRI data extraction}

An axial image at the level of L3 was stored in Digital Imaging and Communications in Medicine (DICOM) format for the CT series (1 image), for the T1-weighted series (1 image) and for the T2-weighted series (1 image). The images were coded and stored in a dedicated folder, where the reader was not able to associate images from the same patient. The set of three images was then uploaded into the Slice-O-Matic software v5.0 (Tomovision, Canada).

* CT semi-automatic segmentation: by using either the morpho mode or the region growing mode, semi-automatic segmentations were drawn by one person with 1 year of experience in imaging evaluation (CZ). In case of doubts regarding segmentation, a senior radiologist with $>15$ years of experience (SR) double-checked the segmentations. The morpho mode uses mathematical morphology to segment and edit the images. Mathematical morphology segmentation is done by computing the watershed of the gradient that gives a mosaiclike appearance to the image. Each region of this mosaic may then be filled with the appropriate tag value, corresponding to the tissue type (mainly muscle, subcutaneous fat and visceral fat). The final step done by the software is to merge the areas of these regions together. CT attenuation thresholds were -29 to 150 Hounsfield units (HU) for skeletal muscle; -190 to $-30 \mathrm{HU}$ for SAT; -150 to $-50 \mathrm{HU}$ for VAT.

* CT automatic segmentation: the Automatic Body composition Analyzer using Computed tomography image Segmentation (ABACS) module, commercially available as adjunctive tool to SliceO-Matic, was used. The algorithm behind ABACS follows a two-step approach: in the first step, a muscle region mask is determined using a templatebased segmentation methodology, wherein a binary template defining an initial shape of the muscle is deformed via non-rigid registration to closely match the muscle region in the binarized version (obtained by thresholding within the muscle -29 to $150 \mathrm{HU}$ range) of the input slice. The deformation process is guided by a statistical shape prior model. This aids the disambiguation of the muscle tissue from the neighboring organs with overlapping $\mathrm{HU}$ ranges, leading to an accurate segmentation of the muscle region mask. The second step involves masking the input slice with the estimated muscle region segmentation and determining the final muscle, subcutaneous, and VAT regions of interest using the corresponding pre-defined $\mathrm{HU}$ ranges for these tissues. Specifically, the pixels within the muscle region mask have attenuation values ranging from -29 to $150 \mathrm{HU}$. The pixels lying 'outside' of the outer boundary of the muscle mask that have attenuation values in the -190 to $-30 \mathrm{HU}$ range, comprise the SAT while the pixels that are 'inside' the interior of the inner of the boundary of the muscle mask and have attenuation values in the -150 to $-50 \mathrm{HU}$ range are used to define the VAT 

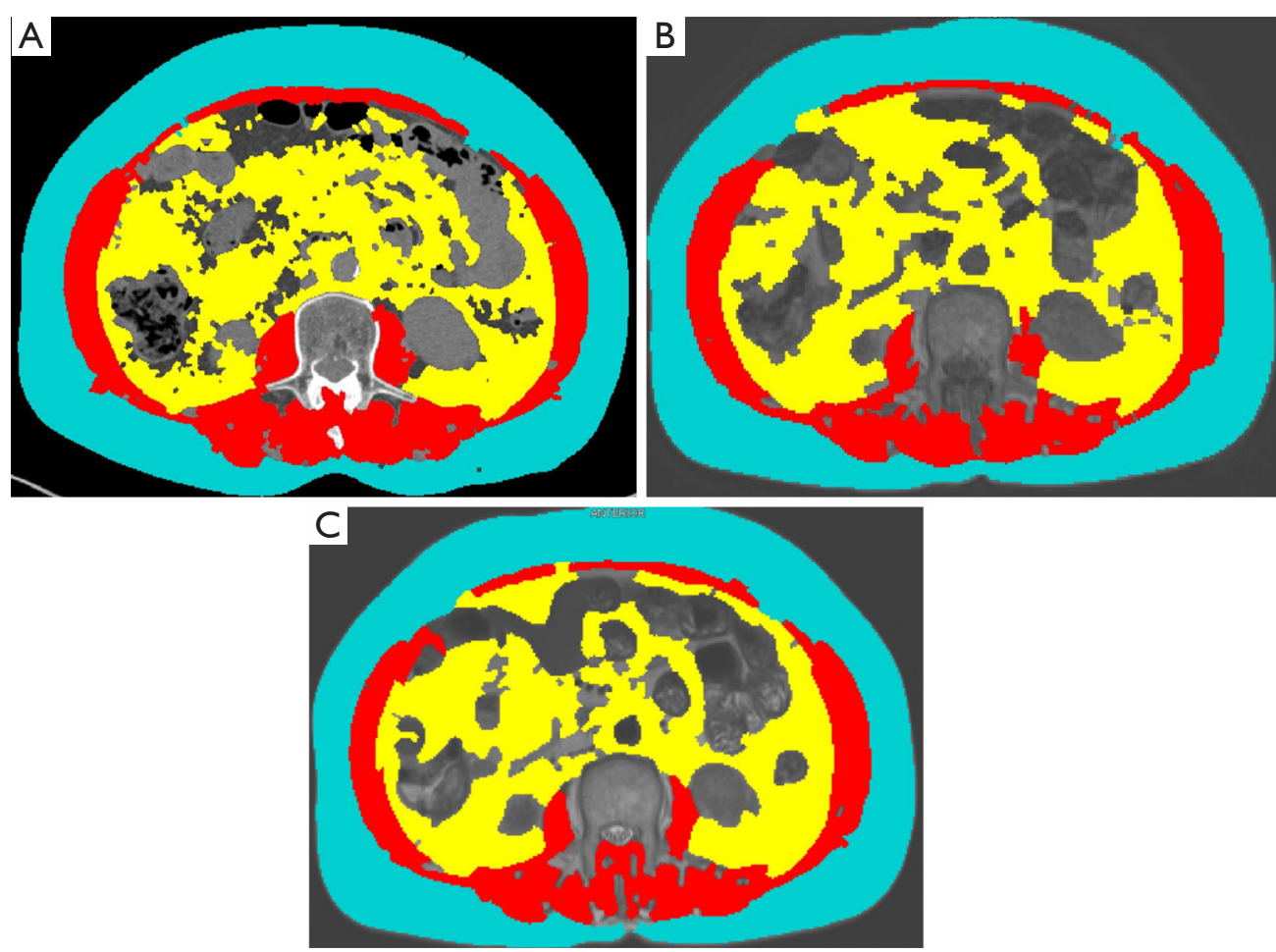

Figure 1 Semi-automatic segmentation of SMA (red) used to calculate SMI, VAT (yellow) and SAT (light blue) from axial images at the level of L3 in the same patients. (A) Non-iodinated CT. (B) MRI T1-weighted sequence. (C) MRI T2-weighted sequence. As shown in this example, main tissues are well depicted on all the images, although some minor differences can be seen within the segmentation of the psoas muscles and abdominal wall (minor missing dots in MRI images), of the SAT (with MRI missing some dots on the surface), and of the VAT (with bowel representing a confounding element). SMA, skeletal muscle area; SMI, skeletal muscle index; VAT, visceral adipose tissue; SAT, subcutaneous adipose tissue; L3, the third lumbar vertebra; CT, computed tomography; MRI, magnetic resonance imaging.

ROI $(20,21)$. The software is available commercially from Voronoi Health Analytics Inc., (Coquitlam, Canada; https://voronoihealthanalytics.com) and is integrated into the SliceOmatic (TomoVision, Magog, Canada; https://tomovision.com) software as a module.

* MRI semi-automatic segmentation: MRI images were segmented by using the morpho mode of Slice-O-Matic.

* By using either the morpho mode or the region growing mode, semi-automatic segmentations were drawn by one person with 1 year of experience in imaging evaluation (CZ). In the case of doubts regarding segmentation, a senior radiologist with $>15$ years of experience (SR) double-checked the segmentations. The following quantitative measurements were recorded: SMA (including the psoas, erector spinae, quadratus lumborum, transversus abdominis, external obliques, internal obliques, and rectus abdominis muscles) expressed in $\mathrm{cm}^{2}$; SAT expressed in $\mathrm{cm}^{2}$; and VAT expressed in $\mathrm{cm}^{2}$. The SMI was then calculated by normalizing SMA by the square height $\left(\mathrm{m}^{2}\right)$ and reported as $\mathrm{cm}^{2} / \mathrm{m}^{2}$. For CT images, the mean values of densities in $\mathrm{HU}$, as reported by the software, were also recorded.

For the set of CT images only, SMA, SAT and VAT were also calculated by using the automatic segmentation.

An example of semiautomatic segmentation of SMA, SAT and VAT on CT and MRI images is shown in Figure 1.

The single axial measures of SAT and VAT were used to calculate the whole body volumes (in liters) of skeletal muscle and VAT, according to the formulas proposed by Shen et al. (6).

\section{Statistical analysis}

Quantitative variables were presented as mean with SD, or 
Table 2 Main characteristics of study population

\begin{tabular}{|c|c|}
\hline Characteristics & Value \\
\hline Age (years), mean $\pm S D$ & $66 \pm 12$ \\
\hline Time interval (days) between the first and the second exam, mean; median; SD & $36.1 ; 31 ; 29.5$ \\
\hline Weight $(\mathrm{kg})$, mean $\pm \mathrm{SD}$ & $70 \pm 15$ \\
\hline Height $(m)$, mean \pm SD & $1.68 \pm 0.11$ \\
\hline BMI $\left(\mathrm{kg} / \mathrm{m}^{2}\right)$, mean $\pm \mathrm{SD}$ & $25 \pm 6.5$ \\
\hline $\mathrm{BSA}\left(\mathrm{m}^{2}\right)$, mean $\pm \mathrm{SD}$ & $1.78 \pm 0.21$ \\
\hline \multicolumn{2}{|l|}{ Clinical indication to perform imaging examinations, $n$ [\%] } \\
\hline Gallbladder and biliary tree lesions & 16 [13] \\
\hline Renal lesions & $13[10]$ \\
\hline Gastro-intestinal cancers & $9[7]$ \\
\hline Other cancers & $9[7]$ \\
\hline
\end{tabular}

*, available in 93/123 patients. SD, standard deviation; BMI, body mass index; BSA, body surface area.

as median with their interquartile ranges, as appropriate. Qualitative data were presented as absolute numbers with percentages.

Quantitative MRI measurements were compared with CT measurements by using Pearson correlation analysis and by Bland-Altman agreement analysis (22). Linear regression analysis explain how the values on the Y-axis vary with the values on the $\mathrm{X}$-axis in a predictable way which can be represented by a straight line. The more the points cluster around the line, the more the model works well and the values on the $\mathrm{Y}$-axis are predictable. In Bland-Altman plots, the central line represents the average difference between methods: the more the points are closer to the central line, the more the differences between the two groups of values are homogeneous and similar to the average difference. Intersperse points show variability in differences between values.

If in the Bland-Altman plots the difference between pairs of measurements increased with the mean, a logarithmic transformation (natural logarithm) of the measurements was performed, as suggested (22). Analysis was performed on total sample, further divided according to BMI and sarcopenia. Different subgroups were built and separately analyzed in order to assess whether these issues could affect the comparison between methods. Furthermore, the following compartments were calculated for the 4 sets of images: (SAT + VAT)/SMI; SAT/VAT; SMI/SAT.

All statistical analyses were carried out with the Stata 15 (StatCorp. LP, College Station, TX, USA).

\section{Results}

According to inclusion and exclusion criteria, 27 patients were excluded and the final cohort consisted of 123 patients (69 males and 54 females) with a mean age of $66 \pm 12$ years, with different clinical indications for undergoing the imaging examinations. Baseline characteristics of the study population are summarized in Table 2. Height (and SMI) were available for $122 / 123$ patients. The mean time between the two time-points was 36.1, and there was no significant difference for time interval $(\mathrm{P}=0.47)$. Weight at the two time-points was available in $93 / 123$ patients, and the mean difference was $-0.2 \mathrm{~kg}(\mathrm{P}=0.9)$. The largest difference in weight between the two time-points was encountered in 3 patients: 1 was due to heart failure (first exam) that was then treated by diuretic therapy $(-12 \mathrm{~kg}) ; 1$ was associated to a large pseudocyst, that was then drained $(-7 \mathrm{~kg}) ; 1$ was associated to a chronic abuse of ethyl $(-10 \mathrm{~kg})$. Details about segmentation values of the 3 outlier patients are included in 
Table 3 Mean and SD of body composition values calculated at L3 level on CT by semi-automatic and automatic segmentation, and on MRI T1-weighted and T2-weighted sequences

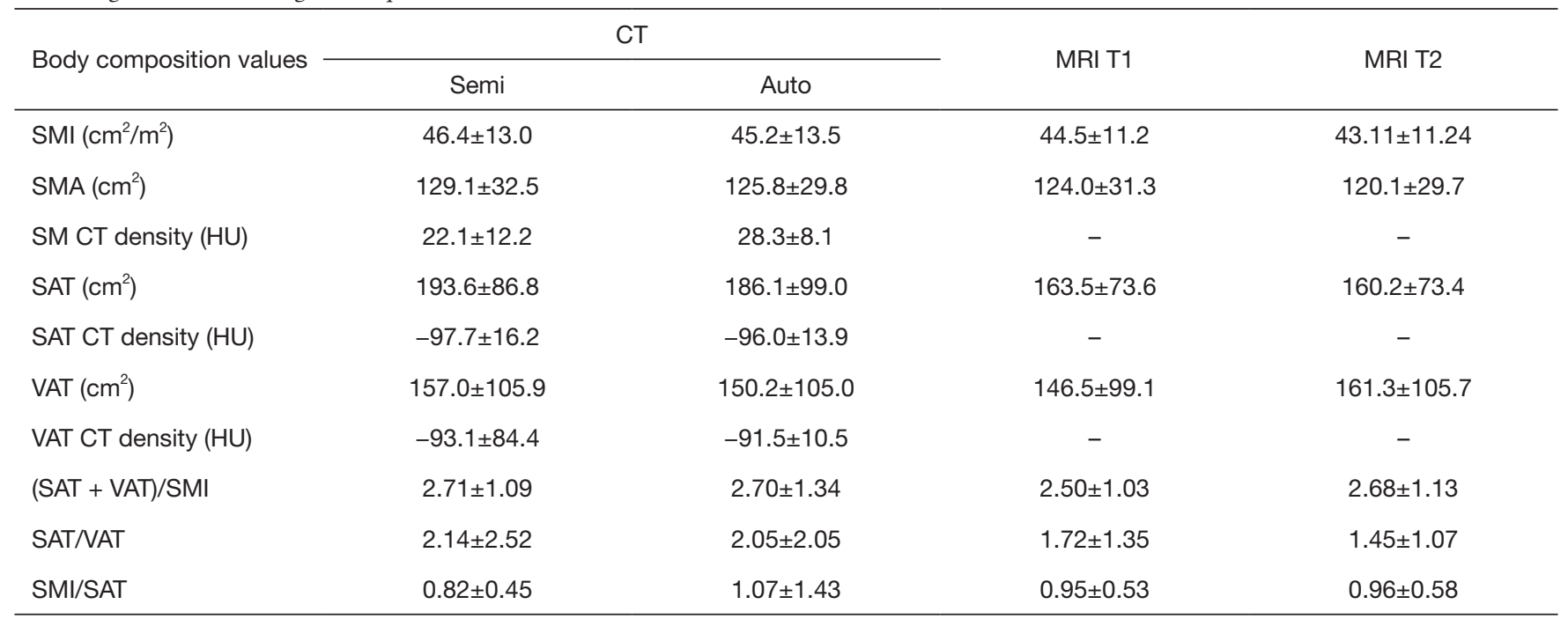

SD, standard deviation; L3, the third lumbar vertebra; CT, computed tomography; MRI, magnetic resonance imaging; SMI, skeletal muscle index; SMA, skeletal muscle area; SM, skeletal muscle; HU, Hounsfield units; SAT, subcutaneous adipose tissue; VAT, visceral adipose tissue.

Table S1.

Assessment of SMI, SAT and VAT areas and their values on CT and MRI are listed in Table 3.

Mean and SD of calculated volumes of SMA from CT images with semiautomatic and automatic segmentations and with MRI on $\mathrm{T} 1$ and $\mathrm{T} 2$ images were $23.6 \pm 5.4 ; 23 \pm 5$; $22.7 \pm 5.2$ and $22.1 \pm 4.9$ liters, respectively. Mean and SD of calculated volumes of VAT from CT images with semiautomatic and automatic segmentations and with MRI on $\mathrm{T} 1$ and $\mathrm{T} 2$ images were $14.8 \pm 7.2 ; 14 \pm 7.3 ; 14.1 \pm 6.8$; $15.1 \pm 7.2$ liters, respectively.

\section{$S M I$}

By performing linear regression analysis, CT and MRI derived measurements of SMI showed a high correlation $\left(r^{2}=0.81\right.$ for $\mathrm{T} 1 ; \mathrm{r}^{2}=0.89$ for T2). The Bland-Altman analysis comparing the SMI calculated from CT and MRI images showed an average difference of 0.041 for T1-weighted images (95\% confidence interval: 0.020 to 0.062 ; limits of agreement: -0.192 to 0.274 ), and 0.072 for T2-weighted images ( $95 \%$ confidence interval: 0.055 to 0.088 ; limits of agreement: -0.109 to 0.252 ), respectively (Figure 2 ).

\section{$S A T$}

By performing linear regression analysis, CT and MRI derived measurements of SAT showed a high correlation $\left(\mathrm{r}^{2}=0.81\right.$ for $\mathrm{T} 1 ; \mathrm{r}^{2}=0.81$ for T2). The Bland-Altman analysis comparing SAT calculated from CT and from MRI images showed an average difference of 0.0174 for T1-weighted images (95\% confidence interval: 0.141 to 0.206 ; limits of agreement: 0.197 to 0.544 ), and 0.201 for $\mathrm{T} 2$-weighted differences ( $95 \%$ confidence interval: 0.167 to 0.236 ; limits of agreement: -0.190 to 0.593 ), respectively (Figure 3).

\section{VAT}

By performing linear regression analysis, CT and MRI derived measurements of SAT showed a high correlation $\left(r^{2}=0.94\right.$ for $T 1 ; r^{2}=0.93$ for T2). The Bland-Altman analysis comparing VAT calculated from CT and from MRI images showed an average difference of 0.040 for T1-weighted images (95\% confidence interval: -0.001 to 0.081 ; limits of agreement: -0.428 to 0.508 ), and -0.084 for $\mathrm{T} 2$-weighted images (95\% confidence interval: -0.131 to -0.036 ; limits of agreement: -0.623 to 0.456 ), respectively (Figure 4).

Assessment of SMI, SAT and VAT values on CT, MRI T1-weighted and MRI T2-weighted images, performed on subgroups derived according to BMI and sarcopenia, are shown in Table 4.

Table 5 shows results of Bland-Altman agreement analysis, comparing MRI measurements and CT measurements for each sub-group. Results are reported as 

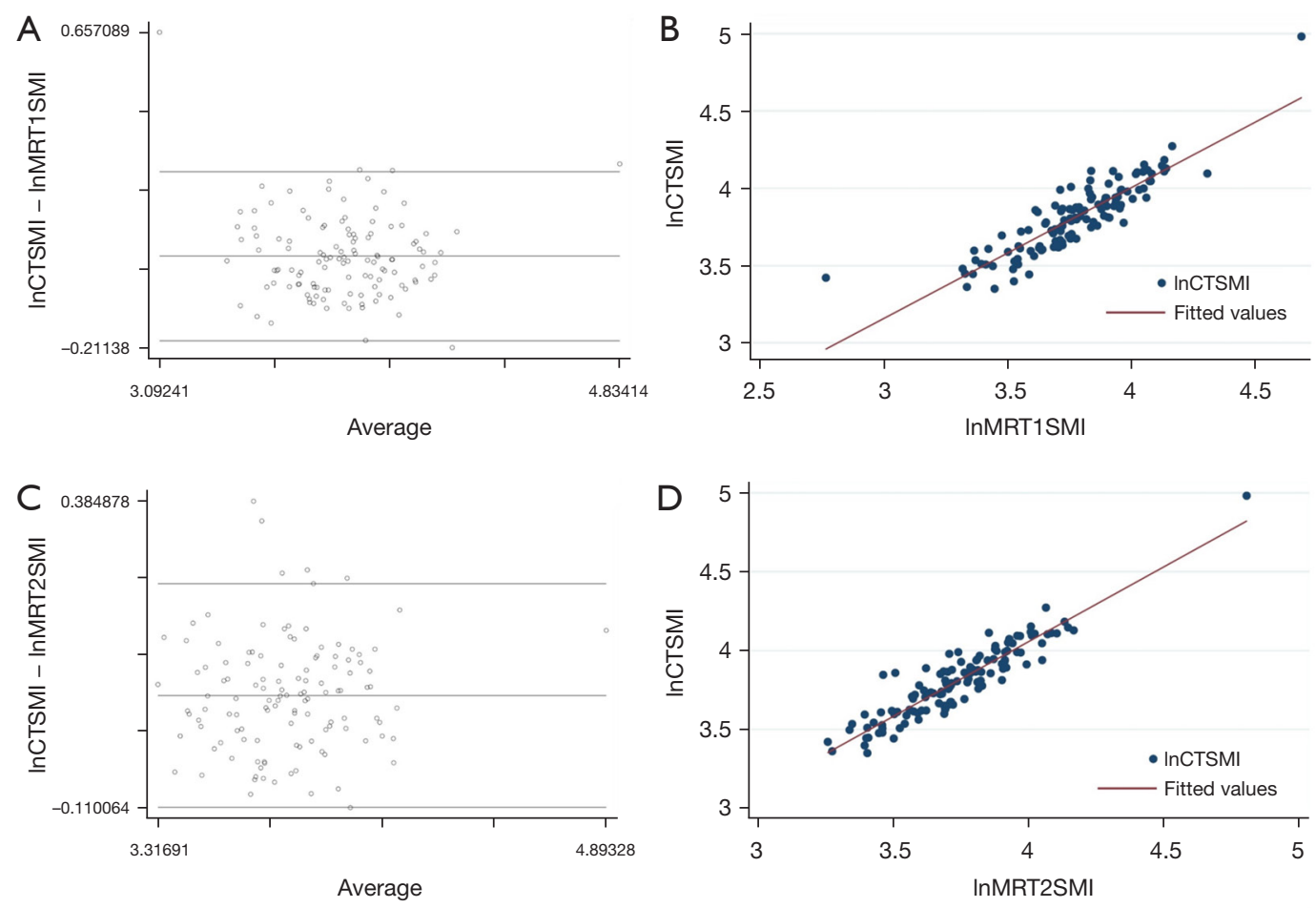

Figure 2 Bland-Altman plots and linear regression analysis, showing the average on the logarithmic scale of the difference between SMI measured by CT and MRI. (A) Bland-Altman plot of lnCTSMI and by MRI T1-weighted (lnMRT1SMI) images. (B) Linear regression analysis of lnCTSMI and lnMRT1SMI images. (C) Bland-Altman plot of lnCTSMI and lnMRT2SMI images. (D) Linear regression analysis of lnCTSMI and by MRI T2-weighted images. The linear regression analysis (B,D) shows a clustering of points around the straight line, demonstrating that the MRI measurements of SMI are predictable starting from the CT measurements. The central line of the BlandAltman plots represents the average difference between the two methods. In this analysis (A,C) most of the dots are equally distributed above and below the central line; moreover the measurements difference is more homogeneous comparing the MRI T1-weighted images to CT images than MRI T2 images to CT images. SMI, skeletal muscle index; CT, computed tomography; MRI, magnetic resonance imaging; lnCTSMI, the difference between SMI measured by CT; lnMRT1SMI, the difference between SMI by MRI T1-weighted; lnMRT2SMI, the difference between SMI by MRI T2-weighted.

average difference with relative $95 \%$ confidence interval and limits of agreement.

\section{CT segmentation}

The comparison of values calculated by using the semiautomatic $v s$. the automatic segmentation method showed differences on the logarithmic scale of 0.025 for SMI (95\% confidence interval: 0.003 to 0.047 ; limits of agreement: -0.221 to 0.272 ); 0.053 for VAT (95\% confidence interval: 0.013 to 0.092 ; limits of agreement: -0.394 to 0.499$)$, and 0.094 for SAT (95\% confidence interval: 0.054 to 0.134 ; limits of agreement: -0.364 to 0.552 ), respectively (Figure 5). An example of semi-automatic and automatic segmentation of SMA, SAT and VAT on the same CT image is shown in Figure 6.

\section{Discussion}

Body composition assessment is gaining increasing attention in many clinical settings, because of its role in metabolism of drugs, advent of therapy complications, cardiovascular and metabolic risk $(4,23)$. Sarcopenia is a condition characterized by loss of muscle mass and muscle strength. Muscle mass decrease is directly responsible for functional impairment with loss of strength, increased likelihood of falls, and loss of autonomy (24). Cachexia is a multifactorial syndrome characterized by severe body weight, fat and 

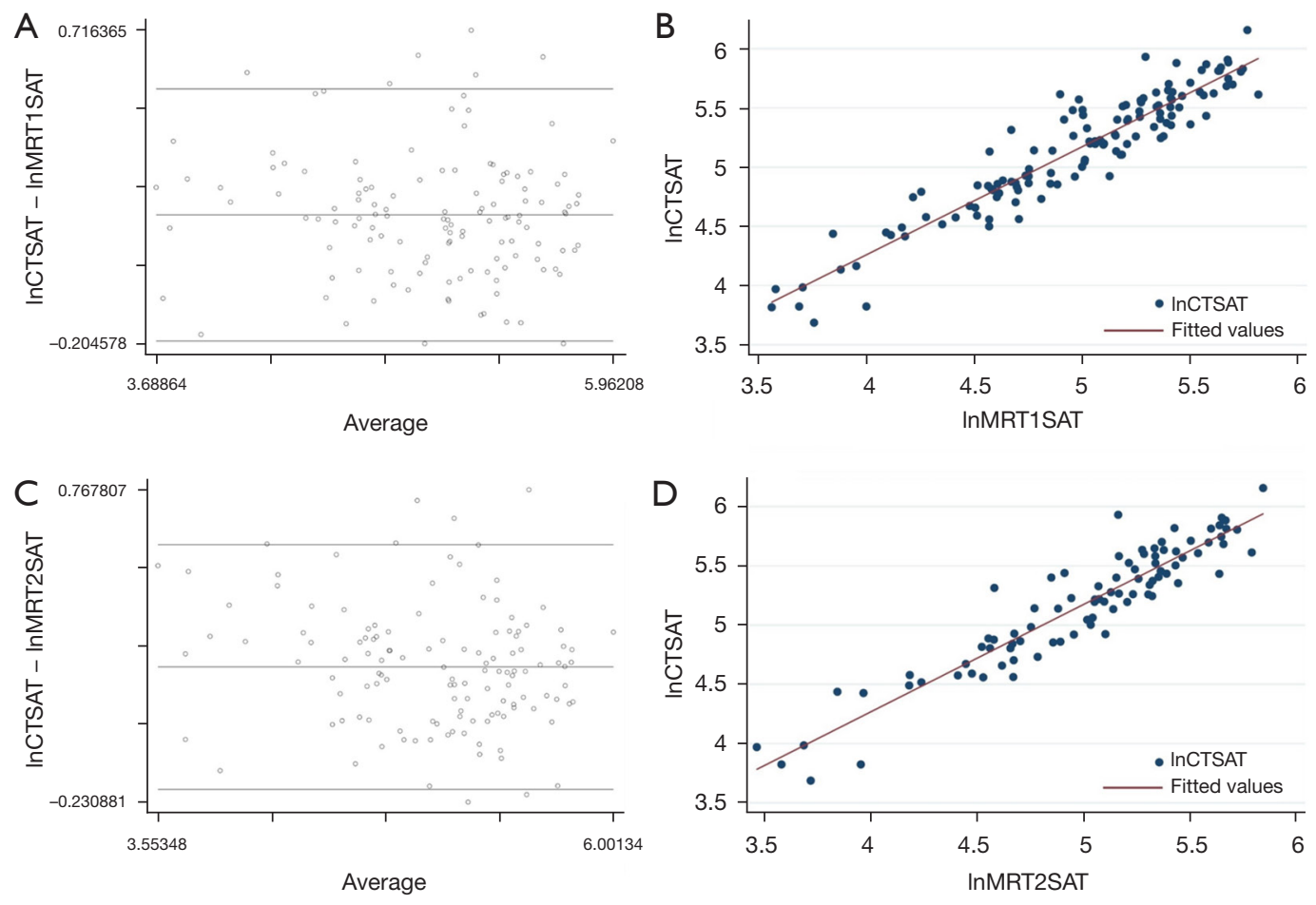

Figure 3 Bland-Altman plots and linear regression analysis, showing the average on the logarithmic scale of the difference between SAT measured by CT and MRI. (A) Bland-Altman plot of lnCTSAT and lnMRT1SAT images. (B) Linear regression analysis of lnCTSAT and lnMRT1SAT images. (C) Bland-Altman plot of lnCTSAT and lnMRT2SAT images. (D) Linear regression analysis of lnCTSAT and lnMRT2SAT images. The linear regression analysis (B,D) shows a clustering of points around the straight line, but shows also some points distant from the central line. Accordingly, the Bland-Altman plots (A,C) show that there are some outliers dots comparing MRI T1-weighted images to CT images as well as comparing MRI T1-weighted images to CT images. SAT, subcutaneous adipose tissue; CT, computed tomography; MRI, magnetic resonance imaging; lnCTSAT, the difference between SAT measured by CT; lnMRT1SAT, the difference between SAT by MRI T1-weighted; lnMRT2SAT, the difference between SAT by MRI T2-weighted.

muscle loss and increased protein catabolism due to underlying diseases; this condition is clinically relevant since it increases patients' morbidity and mortality (24).

The CT scan offers a good evaluation for body composition analysis, thanking its accuracy in quantification of muscle and fat, and its fast acquisition. In the oncological setting, where patients are usually evaluated over time by CT (25-28) a retrospective evaluation of skeletal muscle from clinical CT examinations is indeed an opportunistic source of significant information regarding sarcopenia and fat distribution (23). In these patients CT scan offers also the opportunity to assess the attenuation values of muscle and fat, which give information about the functional status of these tissues. For instance, low attenuation values within the muscle, indicate high fat infiltration and lower performance. However, for some categories of patients, such as patients with pancreatic and hepatic lesions, MRI is frequently used for imaging assessment instead of CT, as demonstrated in this study cohort, where the most frequent indications to perform the exams were evaluations of pancreatic and hepatic lesions. We therefore deemed it important to evaluate whether MRI can be as reliable as CT in evaluation of body composition quantitative values. With this regard, Tandon et al. demonstrated that CT and MRI were comparable in the evaluation of SMI in 61 liver transplant donors, and conclude that MRI can be used interchangeably with CT to quantify L3 skeletal muscle cross-sectional area (29), although in their study there were some significant outliers, mostly favoring the use of CT $(29,30)$. Therefore, the question of whether or not CT and MRI measurements of skeletal muscle are comparable was still open and needed further evaluations. Higgins et al. performed a comparison 

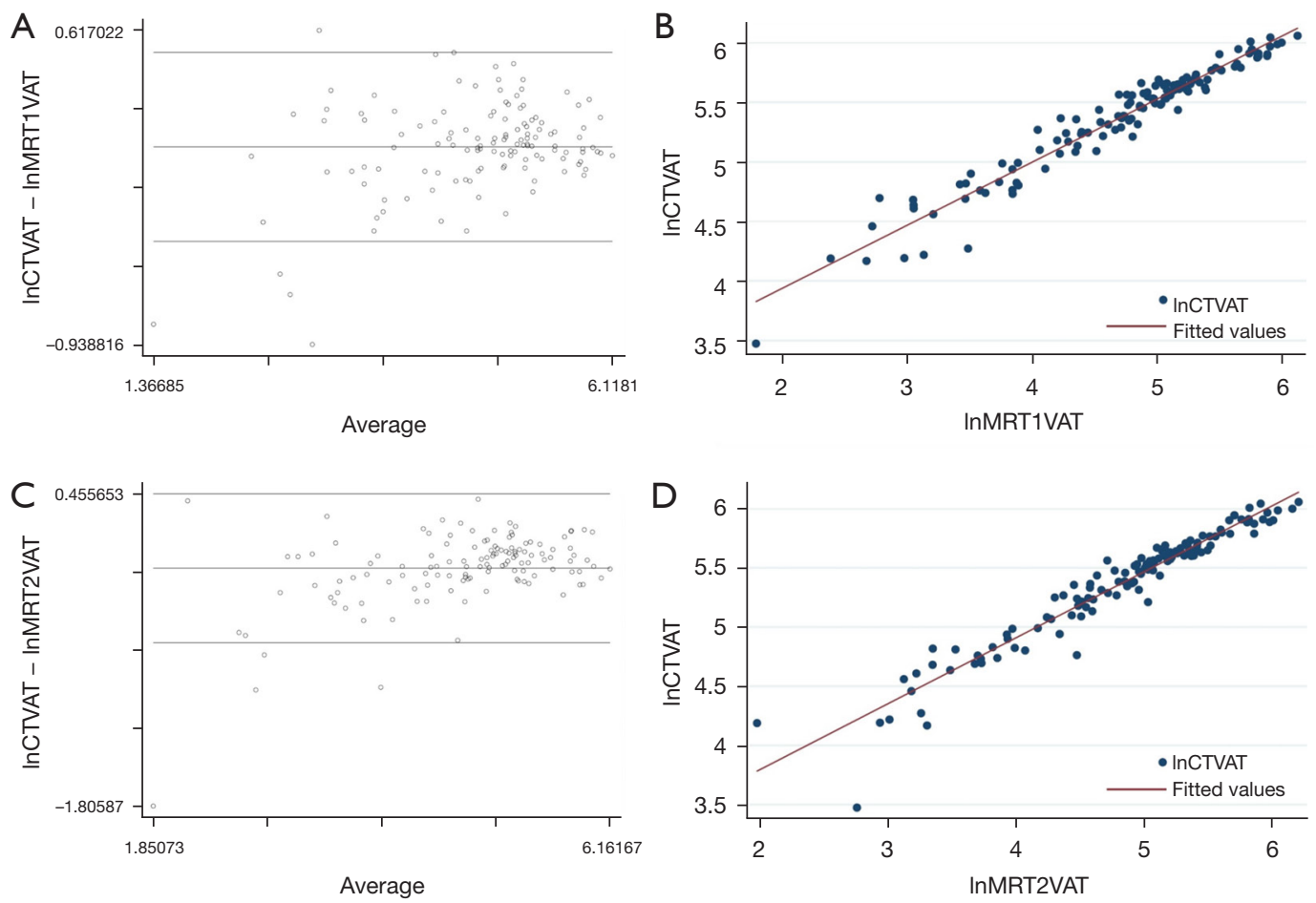

Figure 4 Bland-Altman plots and linear regression analysis, showing the average on logarithmic scale of the difference between VAT measured by CT and by MRI. (A) Bland-Altman plot of lnCTVAT and lnMRT1VAT. (B) Linear regression analysis of lnCTVAT and lnMRT1VAT images. (C) Bland-Altman plot of lnCTVAT and lnMRT2VAT images. (D) Linear regression analysis of lnCTVAT and $\operatorname{lnMRT2VAT}$ images. The linear regression analysis (B,D) shows a clustering of points around the straight line, demonstrating that the MRI measurements of VAT are predictable starting from the CT measurements. The Bland-Altman plots (A,C) show that there are some outliers dots comparing MRI T1-weighted images to CT images as well as comparing MRI T1-weighted images to CT images, being MRI measurements more prone to overestimate the VAT values. VAT, visceral adipose tissue; CT, computed tomography; MRI, magnetic resonance imaging; lnCTVAT, the difference between VAT measured by CT; $\operatorname{lnMRT1VAT}$, the difference between VAT by MRI T1weighted; lnMRT2VAT, the difference between VAT by MRI T2-weighted.

Table 4 Assessment of SMI, SAT and VAT values on CT, MRI T1-weighted and MRI T2-weighted images, performed on subgroups derived according to BMI and sarcopenia

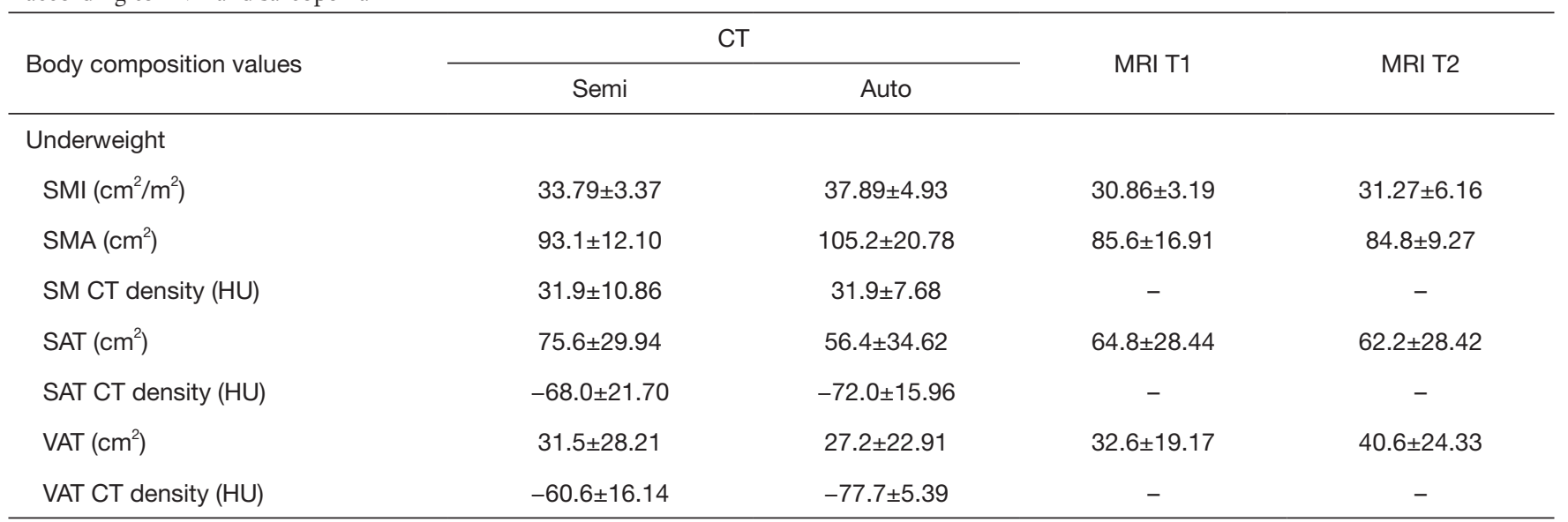

Table 4 (continued) 
Table 4 (continued)

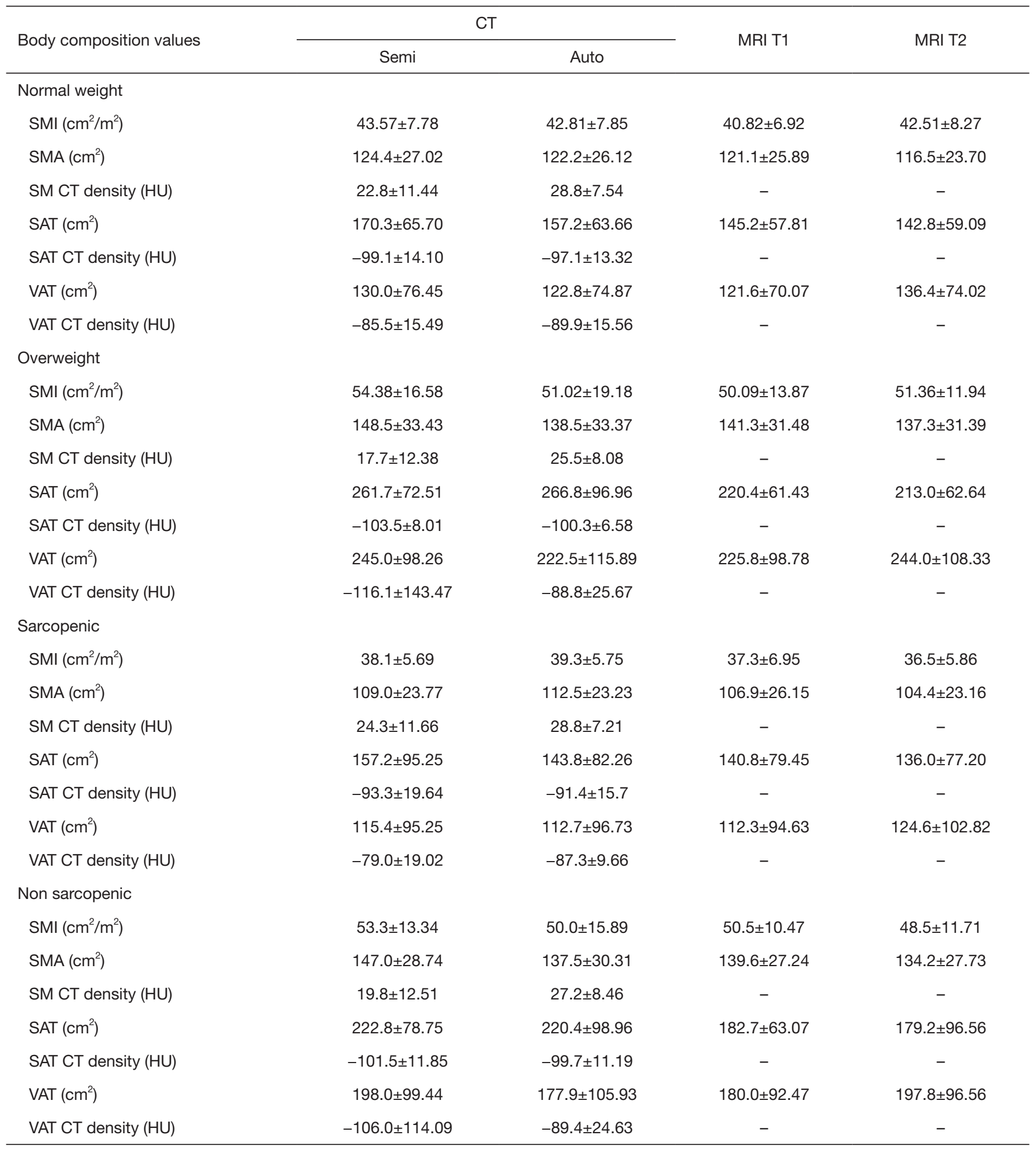

SMI, skeletal muscle index; SAT, subcutaneous adipose tissue; VAT, visceral adipose tissue; CT, computed tomography; MRI, magnetic resonance imaging; BMI, body mass index; SMA, skeletal muscle area; SM, skeletal muscle; HU, Hounsfield units. 
Table 5 Bland-Altman agreement analysis, comparing MRI measurements and CT measurements for each sub-group. Results are reported as average difference with relative $95 \%$ confidence interval and limits of agreement

\begin{tabular}{|c|c|c|c|c|c|c|}
\hline $\begin{array}{l}\text { Body composition } \\
\text { values }\end{array}$ & Total sample & \multicolumn{2}{|c|}{ Muscle condition } & \multicolumn{3}{|c|}{ BMI } \\
\hline \multicolumn{7}{|l|}{ SMI index } \\
\hline \multicolumn{7}{|l|}{ CT vs. T1 } \\
\hline Av. diff. & 0.041 & 0.027 & 0.052 & 0.096 & 0.026 & 0.049 \\
\hline Lim. agr. & -0.192 to 0.274 & -0.227 to 0.282 & -0.162 to 0.265 & -0.312 to 0.504 & -0.178 to 0.233 & -0.147 to 0.245 \\
\hline \multicolumn{7}{|l|}{ CT vs. T2 } \\
\hline Av. diff. & 0.072 & 0.044 & 0.094 & 0.091 & 0.063 & 0.080 \\
\hline Conf. int. & 0.055 to 0.088 & 0.023 to 0.065 & 0.071 to 0.117 & 0.037 to 0.145 & 0.041 to 0.086 & 0.051 to 0.107 \\
\hline \multicolumn{7}{|l|}{ SAT } \\
\hline \multicolumn{7}{|l|}{ CT vs. T1 } \\
\hline Av. diff. & 0.174 & 0.138 & 0.198 & 0.150 & 0.170 & 0.174 \\
\hline Conf. int. & 0.141 to 0.206 & 0.084 to 0.192 & 0.156 to 0.240 & 0.011 to 0.289 & 0.126 to 0.213 & 0.123 to 0.225 \\
\hline Lim. agr. & 0.197 to 0.544 & -0.262 to 0.537 & -0.146 to 0.542 & -0.310 to 0.611 & -0.198 to 0.538 & -0.163 to 0.511 \\
\hline \multicolumn{7}{|l|}{ CT vs. T2 } \\
\hline Av. diff. & 0.201 & 0.179 & 0.223 & 0.200 & 0.194 & 0.211 \\
\hline Conf. int. & 0.167 to 0.236 & 0.123 to 0.236 & 0.176 to 0.269 & 0.065 to 0.335 & 0.148 to 0.240 & 0.152 to 0.270 \\
\hline Lim. agr. & 0.190 to 0.593 & -0.236 to 0.595 & -0.160 to 0.605 & -0.245 to 0.646 & -0.192 to 0.580 & -0.179 to 0.602 \\
\hline \multicolumn{7}{|l|}{ CT vs. T2 } \\
\hline Av. diff. & -0.084 & -0.165 & -0.014 & -0.312 & -0.097 & 0.016 \\
\hline Conf. int. & -0.131 to -0.036 & -0.256 to -0.073 & -0.056 to 0.027 & -0.548 to -0.076 & -0.160 to -0.435 & -0.029 to 0.061 \\
\hline Lim. agr. & -0.623 to 0.456 & -0.840 to 0.511 & -0.356 to 0.327 & -1.092 to 0.469 & -0.623 to 0.456 & -0.282 to 0.315 \\
\hline
\end{tabular}

MRI, magnetic resonance imaging; CT, computed tomography; SMI, skeletal muscle index; av. diff., average difference; conf. int., confidence interval; lim. agr., limits of agreement; SAT, subcutaneous adipose tissue; VAT, visceral adipose tissue.

of body composition values in 58 patients who underwent CT and MRI as pre-operative assessment for renal cell carcinoma. The authors demonstrated a strong correlation of measurements of skeletal muscle and adipose tissues, thus supporting the use of axial MRI images for comprehensive measurement of body composition (31). Khan et al. assessed the reproducibility and concordance of CT and MRI segmentation analyses of SMA at mid-L3 on a small sample of 9 patients with renal cell carcinoma, demonstrating a strong correlation of SMA at mid-L3 between CT and T2- 

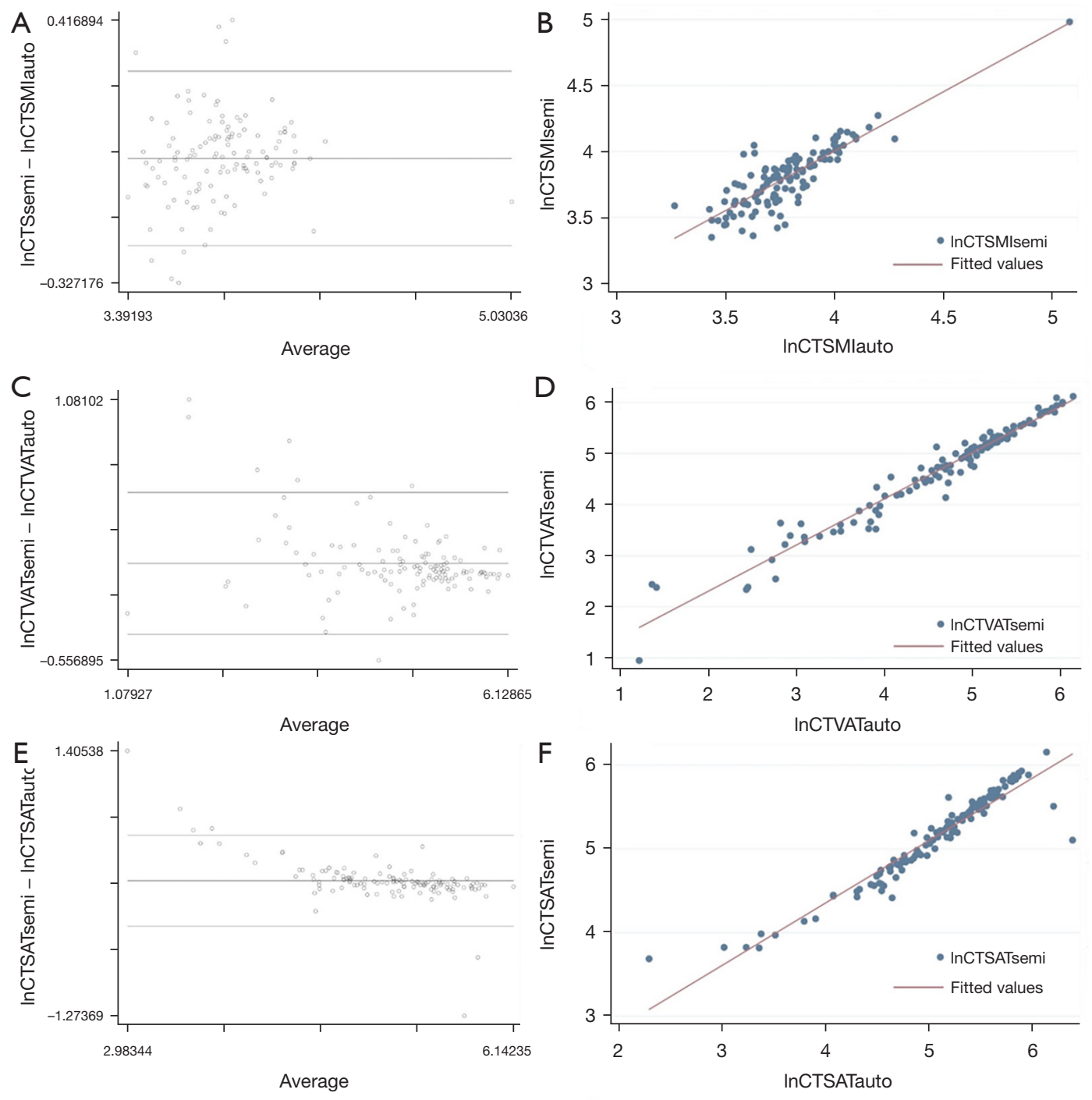

Figure 5 Bland-Altman plots and linear regression analysis, showing the average on the logarithmic scale of the difference between computed tomography (CT) values extracted by semi-automatic segmentation and automatic segmentation. (A) Bland-Altman plot for SMI (lnCTSMIsemi - lnCTSMIauto). (B) Linear regression analysis for SMI. (C) Bland-Altman plot for VAT (lnCTVATsemi - lnCTVATauto). (D) Linear regression analysis for VAT. (E) Bland-Altman plot for SAT (lnCTSATsemi - lnCTSATauto). (F) Linear regression analysis for SAT. CT, computed tomography; SMI, skeletal muscle index; lnCTSMI, the difference between SMI measured by CT; VAT, visceral adipose tissue; lnCTVAT, the difference between VAT measured by CT; SAT, subcutaneous adipose tissue; lnCTSAT, the difference between SAT measured by CT.

weighted MRI (32).

Wang et al. assessed the validity and reliability of abdominal SMA measurement by comparing CT and MRI based on the fat image of IDEAL-IQ sequence at the lumbar level mid-L3, demonstrating that MRI exhibited good interobserver reliability and excellent agreement with CT for quantification of abdominal SMA (33). Sinelnikov et al. investigated the correlation between CT and MR measurements of SMA in the abdomen of 12 patients, and demonstrated that SMA at CT correlated closely with MR, with $\mathrm{T} 2$-weighted images showing the highest correlation $(\mathrm{r}=0.98 ; \mathrm{P}<0.01)(34)$. According to the abovementioned studies, in a larger cohort of 123 subjects, we demonstrated that SMI, derived from SMA, measured by CT and MRI at 

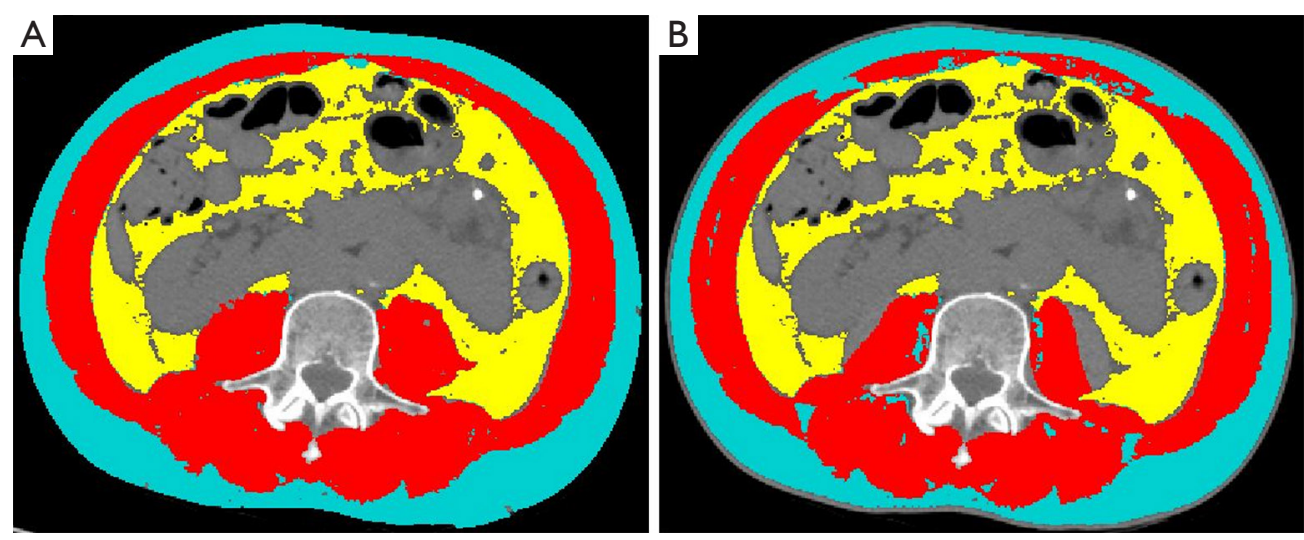

Figure 6 SMA (red) used to calculate SMI, VAT (yellow) and SAT (light blue) on the same CT image. (A) Segmentation performed by semiautomatic tool. (B) segmentation performed by automatic tool. In this example, the automatic segmentation failed to include some small muscle areas (as the lateral part of the psoas muscles and some small dots within the anterior abdominal wall), likely due to low densities related to fat infiltration. SMA, skeletal muscle area; SMI, skeletal muscle index; VAT, visceral adipose tissue; SAT, subcutaneous adipose tissue; CT, computed tomography.

the level of L3 showed a high correlation $\left(\mathrm{r}^{2}=0.81\right.$ for $\mathrm{T} 1$; $\mathrm{r}^{2}=0.89$ for $\mathrm{T} 2$ ).

The interchangeability of the two techniques would be of great help when the same patient undergoes CT and MRI at different time-points, also for the assessment of fat distribution. Indeed, the variation of VAT over time may help to evaluate patients after bariatric surgery (35), or under dietary restrictions (36), or included in weight loss programs, or under physical exercise regimens (15). In a study focused on 31 patients, accuracy of MRI measurement of VAT was compared to CT and the authors demonstrated an accuracy of T1-weighted and T2-weighted MRI of $89 \%$ and $91 \%$ compared to CT (37). CT and MRI have both been used as tools to investigate the distribution of SAT and VAT $(38,39)$. If CT offers an opportunistic evaluation of fat infiltration by attenuation values, MRI using Dixon technique may give information as reliable. However, Dixon sequences have to be planned in advance, and for the calculation of fat infiltration, both the water-only and the fat-only should be available on the PACS. In our cohort of 123 patients, the VAT measurements by MRI and CT showed a high correlation, both with T1-weighted images, and with $\mathrm{T} 2$-weighted images, with an average difference of 0.040 for $\mathrm{T} 1$-weighted images and -0.084 for $\mathrm{T} 2$ weighted images. This good performance of quantification from both sequences is likely due to the semi-automatic segmentation allowing selection and exclusion of the intrabowel hyperintensity, which may fall in the same grey level intensities of fat using an automatic segmentation method. Nonetheless, looking at the Bland-Altman plots, the lowest median difference in VAT assessment, was shown by the comparison of MRI T2-weighted images and CT images, being T2-weighted images more prone to overestimate VAT. This difference may be due to the intrinsic characteristic of the $\mathrm{T} 2$-weighted images, where the hyperintensity of fat within the visceral cavity may allow an easier identification for the radiologist's eye compared to T1-weighted images and non-contrast CT images. This is indeed confirmed by the assessment after division in subgroups according to BMI, where the overestimation of T2weighted images is more evident in underweight patients.

Despite its prognostic value, body composition is rarely assessed as a default to improve risk stratification before surgery and chemotherapy, or to personalize lifestyle interventions during cancer therapy. This may be due to a lack of time-efficient, clinic-friendly assessment tools that produce accurate muscle and adipose tissue quantifications. Automated analysis of body composition has the potential to substantially reduce this workload and to accelerate research in body composition. For this reason, we compared the results in body composition assessment of a semi-automatic and automatic segmentation.

In our cohort, the two segmentation methods showed high agreement of the measurements. This is in accordance with the results of Cespedes Feliciano et al., who in a large cohort of 5,990 patients demonstrated a strong agreement between manual and automatic segmentations overall, as well as within subgroups of age, sex, BMI, 
and cancer stage, although the automatic segmentation performance was lowest for some patients $(<2 \%)$ who were underweight or had anatomic abnormalities (20). It is probable that we did not encounter this finding because one of our exclusion criteria was the presence of extensive artifacts or bowel motion, which can alter the segmentation (40), particularly with the automatic tool. In a recent study, Dalah et al. compared automatic and semiautomatic segmentation of VAT and SAT determined on T2-weighted MRI in 55 overweight/obese adults, demonstrating that semi-automatic segmentation showed a stronger correlation with bioelectrical impedance analysis, compared to manual segmentation (41). Therefore, although automatic segmentations are correlated with semi-automatic segmentations and can be used as well, it may be important to consider a visual check of the images before segmentation, mainly when using the automatic segmentation.

This study has some limitations. First, the selection of patients was not based on the clinical indication, but on the availability of CT and MR scans of the same patient. This might have affected the typology of patients selected, as demonstrated by the high number of oncological patients. Oncological patients may be prone to rapid changes in body composition for disease-related problems, such as cachexia, as well as for therapy-related complications (nausea, vomiting, etc.). However, we restricted the period for the inclusion of the two examinations to 3 months, specifically to reduce the possibility of major changes in body composition, even in oncological patients; furthermore, we evaluated the changes in weight between the two exams, when this data was available. Second, we did not assess any inter-reader agreement. However, since the primary objective of the study was to assess differences in quantitative measurements between two different imaging modalities, we tried to keep all the other variables as constant as possible, including the number of readers. Third, we could not perform a comparison of MRI measurements made with more sophisticated evaluations, such as fat-referenced acquisitions. However, this type of assessment, which shows great promise for a precise quantification of body composition and the related risks, needs dedicated acquisition and can therefore be included only in a prospective way. Since the main objective of this study was to assess whether MRI and CT quantitative measures of body composition assessed at the level of L3 are comparable for use in retrospective studies, a prospective acquisition was beyond our scope. Furthermore, we do know that body composition analysis may rely on changes of organ/tissue areas overtime, and not only on a single time evaluation. Therefore, in a more comprehensive evaluation of patients, the assessment of body composition at the level of L3, as done in this study, may not reflect changes at the whole body level (42). Nevertheless, in such evaluations, additional assessments may be planned in advance, according to the clinical setting under evaluation.

Finally, we did include examination over a large time span and this may potentially influence the quality of segmentation; nevertheless, it is known that contrast medium may influence the quality of segmentation (16) and all the CT scans were performed without contrast medium.

In conclusion, our results demonstrate that quantification of SMI, SAT and VAT at MRI from T1-weighted and T2weighted images, is correlated to corresponding values at CT. Therefore, these results support the use of either CT or MRI to quantify body composition parameters, irrespective of the intrinsic physical differences between them. Eventually, on one hand this will allow not to exclude assessments in retrospective studies where either one is present at a pre-determined time point, on the other, in prospective studies, it will avoid patients undergoing unnecessary additional exams, since either one can be performed, according to clinical indication.

Furthermore, semi-automatic and automatic quantification of body composition values at $\mathrm{CT}$ are correlated, but a visual check of images is advisable, especially when using automatic segmentation.

\section{Acknowledgments}

We thank Dr. Susan J. West's help for the language editing. Funding: None.

\section{Footnote}

Reporting Checklist: The authors have completed the MDAR checklist. Available at https://dx.doi.org/10.21037/ qims-21-619

Conflicts of Interest: All authors have completed the ICMJE uniform disclosure form (available at https://dx.doi. org/10.21037/qims-21-619). The authors have no conflicts of interest to declare.

Ethical Statement: The authors are accountable for all 
aspects of the work in ensuring that questions related to the accuracy or integrity of any part of the work are appropriately investigated and resolved. The study was conducted in accordance with the Declaration of Helsinki (as revised in 2013). The study was approved by regional ethics committee of Canton Ticino and individual consent for this retrospective analysis was waived.

Open Access Statement: This is an Open Access article distributed in accordance with the Creative Commons Attribution-NonCommercial-NoDerivs 4.0 International License (CC BY-NC-ND 4.0), which permits the noncommercial replication and distribution of the article with the strict proviso that no changes or edits are made and the original work is properly cited (including links to both the formal publication through the relevant DOI and the license). See: https://creativecommons.org/licenses/by-nc-nd/4.0/.

\section{References}

1. Yip C, Dinkel C, Mahajan A, Siddique M, Cook GJ, Goh $\mathrm{V}$. Imaging body composition in cancer patients: visceral obesity, sarcopenia and sarcopenic obesity may impact on clinical outcome. Insights Imaging 2015;6:489-97.

2. Prado CM, Baracos VE, McCargar LJ, Mourtzakis M, Mulder KE, Reiman T, Butts CA, Scarfe AG, Sawyer MB. Body composition as an independent determinant of 5-fluorouracil-based chemotherapy toxicity. Clin Cancer Res 2007;13:3264-8.

3. Thibault R, Genton L, Pichard C. Body composition: why, when and for who? Clin Nutr 2012;31:435-47.

4. Borga M, West J, Bell JD, Harvey NC, Romu T, Heymsfield SB, Dahlqvist Leinhard O. Advanced body composition assessment: from body mass index to body composition profiling. J Investig Med 2018;66:1-9.

5. Mitsiopoulos N, Baumgartner RN, Heymsfield SB, Lyons W, Gallagher D, Ross R. Cadaver validation of skeletal muscle measurement by magnetic resonance imaging and computerized tomography. J Appl Physiol (1985) 1998;85:115-22.

6. Shen W, Punyanitya M, Wang Z, Gallagher D, St-Onge MP, Albu J, Heymsfield SB, Heshka S. Total body skeletal muscle and adipose tissue volumes: estimation from a single abdominal cross-sectional image. J Appl Physiol (1985) 2004;97:2333-8.

7. Shachar SS, Williams GR, Muss HB, Nishijima TF. Prognostic value of sarcopenia in adults with solid tumours: A meta-analysis and systematic review. Eur J
Cancer 2016;57:58-67.

8. Prado CM, Cushen SJ, Orsso CE, Ryan AM. Sarcopenia and cachexia in the era of obesity: clinical and nutritional impact. Proc Nutr Soc 2016;75:188-98.

9. Prado CM, Birdsell LA, Baracos VE. The emerging role of computerized tomography in assessing cancer cachexia. Curr Opin Support Palliat Care 2009;3:269-75.

10. Fox CS, Massaro JM, Hoffmann U, Pou KM, MaurovichHorvat P, Liu CY, Vasan RS, Murabito JM, Meigs JB, Cupples LA, D'Agostino RB Sr, O'Donnell CJ. Abdominal visceral and subcutaneous adipose tissue compartments: association with metabolic risk factors in the Framingham Heart Study. Circulation 2007;116:39-48.

11. Goran MI, Bergman RN, Gower BA. Influence of total vs. visceral fat on insulin action and secretion in African American and white children. Obes Res 2001;9:423-31.

12. Liu J, Fox CS, Hickson DA, May WD, Hairston KG, Carr JJ, Taylor HA. Impact of abdominal visceral and subcutaneous adipose tissue on cardiometabolic risk factors: the Jackson Heart Study. J Clin Endocrinol Metab 2010;95:5419-26.

13. Huber FA, Del Grande F, Rizzo S, Guglielmi G, Guggenberger R. MRI in the assessment of adipose tissues and muscle composition: how to use it. Quant Imaging Med Surg 2020;10:1636-49.

14. Brennan DD, Whelan PF, Robinson K, Ghita O, O'Brien JM, Sadleir R, Eustace SJ. Rapid automated measurement of body fat distribution from whole-body MRI. AJR Am J Roentgenol 2005;185:418-23.

15. Machann J, Thamer C, Schnoedt B, Haap M, Haring HU, Claussen CD, Stumvoll M, Fritsche A, Schick F. Standardized assessment of whole body adipose tissue topography by MRI. J Magn Reson Imaging 2005;21:455-62.

16. Gohmann RF, Gottschling S, Seitz P, Temiz B, Krieghoff C, Lücke C, Horn M, Gutberlet M. 3D-segmentation and characterization of visceral and abdominal subcutaneous adipose tissue on CT: influence of contrast medium and contrast phase. Quant Imaging Med Surg 2021;11:697-705.

17. Du Bois D, Du Bois EF. A formula to estimate the approximate surface area if height and weight be known. 1916. Nutrition 1989;5:303-11; discussion 312-3.

18. Obesity: preventing and managing the global epidemic. Report of a WHO consultation. World Health Organ Tech Rep Ser 2000;894:i-xii, 1-253.

19. Martin L, Birdsell L, Macdonald N, Reiman T, Clandinin MT, McCargar LJ, Murphy R, Ghosh S, Sawyer MB, Baracos VE. Cancer cachexia in the age of obesity: 
skeletal muscle depletion is a powerful prognostic factor, independent of body mass index. J Clin Oncol 2013;31:1539-47.

20. Cespedes Feliciano EM, Popuri K, Cobzas D, Baracos VE, Beg MF, Khan AD, Ma C, Chow V, Prado CM, Xiao J, Liu V, Chen WY, Meyerhardt J, Albers KB, Caan BJ. Evaluation of automated computed tomography segmentation to assess body composition and mortality associations in cancer patients. J Cachexia Sarcopenia Muscle 2020;11:1258-69.

21. Cespedes Feliciano EM, Chen WY, Lee V, Albers KB, Prado CM, Alexeeff S, Xiao J, Shachar SS, Caan BJ. Body Composition, Adherence to Anthracycline and TaxaneBased Chemotherapy, and Survival After Nonmetastatic Breast Cancer. JAMA Oncol 2020;6:264-70.

22. Bland JM, Altman DG. Measuring agreement in method comparison studies. Stat Methods Med Res 1999;8:135-60.

23. Pickhardt PJ, Graffy PM, Perez AA, Lubner MG, Elton DC, Summers RM. Opportunistic Screening at Abdominal CT: Use of Automated Body Composition Biomarkers for Added Cardiometabolic Value. Radiographics 2021;41:524-42.

24. Muscaritoli M, Anker SD, Argilés J, Aversa Z, Bauer JM, Biolo G, Boirie Y, Bosaeus I, Cederholm T, Costelli P, Fearon KC, Laviano A, Maggio M, Rossi Fanelli F, Schneider SM, Schols A, Sieber CC. Consensus definition of sarcopenia, cachexia and pre-cachexia: joint document elaborated by Special Interest Groups (SIG) "cachexiaanorexia in chronic wasting diseases" and "nutrition in geriatrics". Clin Nutr 2010;29:154-9.

25. Bellomi M, Rizzo S, Travaini LL, Bazzi L, Trifirò G, Zampino MG, Radice D, Paganelli G. Role of multidetector CT and FDG-PET/CT in the diagnosis of local and distant recurrence of resected rectal cancer. Radiol Med 2007;112:681-90.

26. Monfardini L, Preda L, Aurilio G, Rizzo S, Bagnardi V, Renne G, Maccagnoni S, Vigna PD, Davide D, Bellomi M. CT-guided bone biopsy in cancer patients with suspected bone metastases: retrospective review of 308 procedures. Radiol Med 2014;119:852-60.

27. Petrella F, Rizzo S, Radice D, Borri A, Galetta D, Gasparri R, Solli P, Veronesi G, Bellomi M, Spaggiari L. Predicting prolonged air leak after standard pulmonary lobectomy: computed tomography assessment and risk factors stratification. Surgeon 2011;9:72-7.

28. Rizzo S, Radice D, Femia M, De Marco P, Origgi D, Preda L, Barberis M, Vigorito R, Mauri G, Mauro A, Bellomi M. Metastatic and non-metastatic lymph nodes: quantification and different distribution of iodine uptake assessed by dual-energy CT. Eur Radiol 2018;28:760-9.

29. Tandon P, Mourtzakis M, Low G, Zenith L, Ney M, Carbonneau M, Alaboudy A, Mann S, Esfandiari N, Ma M. Comparing the Variability Between Measurements for Sarcopenia Using Magnetic Resonance Imaging and Computed Tomography Imaging. Am J Transplant 2016;16:2766-7.

30. van Vugt JL, Levolger S, Metselaar HJ, IJzermans JN. Reply to: Comparing the Variability Between Measurements for Sarcopenia Using Magnetic Resonance Imaging and Computed Tomography Imaging. Am J Transplant 2016;16:2768.

31. Higgins MI, Martini DJ, Patil DH, Steele S, Evans S, Petrinec BP, Psutka SP, Sekhar A, Bilen MA, Master VA. Quantification of body composition in renal cell carcinoma patients: Comparing computed tomography and magnetic resonance imaging measurements. Eur J Radiol 2020;132:109307.

32. Khan AI, Reiter DA, Sekhar A, Sharma P, Safdar NM, Patil DH, Psutka SP, Small WC, Bilen MA, Ogan K, Master VA. MRI quantitation of abdominal skeletal muscle correlates with CT-based analysis: implications for sarcopenia measurement. Appl Physiol Nutr Metab 2019;44:814-9.

33. Wang FZ, Sun H, Zhou J, Sun LL, Pan SN. Reliability and Validity of Abdominal Skeletal Muscle Area Measurement Using Magnetic Resonance Imaging. Acad Radiol 2021;28:1692-8.

34. Sinelnikov A, Qu C, Fetzer DT, Pelletier JS, Dunn MA, Tsung A, Furlan A. Measurement of skeletal muscle area: Comparison of CT and MR imaging. Eur J Radiol 2016;85:1716-21.

35. Klein S, Fontana L, Young VL, Coggan AR, Kilo C, Patterson BW, Mohammed BS. Absence of an effect of liposuction on insulin action and risk factors for coronary heart disease. N Engl J Med 2004;350:2549-57.

36. Cordes C, Dieckmeyer M, Ott B, Shen J, Ruschke S, Settles M, Eichhorn C, Bauer JS, Kooijman H, Rummeny EJ, Skurk T, Baum T, Hauner H, Karampinos DC. MRdetected changes in liver fat, abdominal fat, and vertebral bone marrow fat after a four-week calorie restriction in obese women. J Magn Reson Imaging 2015;42:1272-80.

37. Pescatori LC, Savarino E, Mauri G, Silvestri E, Cariati M, Sardanelli F, Sconfienza LM. Quantification of visceral adipose tissue by computed tomography and magnetic resonance imaging: reproducibility and accuracy. Radiol Bras 2019;52:1-6. 
38. Baumgartner RN, Heymsfield SB, Roche AF, Bernardino M. Abdominal composition quantified by computed tomography. Am J Clin Nutr 1988;48:936-45.

39. Shen W, Wang Z, Punyanita M, Lei J, Sinav A, Kral JG, Imielinska C, Ross R, Heymsfield SB. Adipose tissue quantification by imaging methods: a proposed classification. Obes Res 2003;11:5-16.

40. Dalal T, Kalra MK, Rizzo SM, Schmidt B, Suess C, Flohr T, Blake MA, Saini S. Metallic prosthesis: technique to avoid increase in CT radiation dose with automatic tube current modulation in a phantom and patients. Radiology

Cite this article as: Zaffina C, Wyttenbach R, Pagnamenta A, Grasso RF, Biroli M, Del Grande F, Rizzo S. Body composition assessment: comparison of quantitative values between magnetic resonance imaging and computed tomography. Quant Imaging Med Surg 2022;12(2):1450-1466. doi: 10.21037/qims-21-619
2005;236:671-5.

41. Dalah E, Hasan H, Madkour M, Obaideen A, Faris MA. Assessing visceral and subcutaneous adiposity using segmented T2-MRI and multi-frequency segmental bioelectrical impedance: A sex-based comparative study. Acta Biomed 2021;92:e2021078.

42. Schweitzer L, Geisler C, Pourhassan M, Braun W, Glüer CC, Bosy-Westphal A, Müller MJ. What is the best reference site for a single MRI slice to assess whole-body skeletal muscle and adipose tissue volumes in healthy adults? Am J Clin Nutr 2015;102:58-65. 


\section{Supplementary}

Table S1 Segmentation values for the 3 outlier patients, affected by cardiac failure $(n=1)$, pancreatic psudocyst ( $n=2)$, chronic abuse of ethyl ( $n=3$ )

\begin{tabular}{|c|c|c|c|c|}
\hline \multirow{2}{*}{ Body composition values } & \multicolumn{2}{|c|}{ CT } & \multirow{2}{*}{ MRI T1 } & \multirow{2}{*}{ MRI T2 } \\
\hline & Semi & Auto & & \\
\hline \multicolumn{5}{|l|}{ Outlier 1} \\
\hline $\mathrm{SMA}\left(\mathrm{cm}^{2}\right)$ & 100.7 & 94.8 & 104.5 & 110 \\
\hline SMA CT density (HU) & 9.8 & 18.16 & - & - \\
\hline SAT CT density (HU) & -114.6 & -103 & - & - \\
\hline VAT $\left(\mathrm{cm}^{2}\right)$ & 190.4 & 187.7 & 216.7 & 192.1 \\
\hline VAT CT density (HU) & -89.2 & -91.8 & - & - \\
\hline \multicolumn{5}{|l|}{ Outlier 2} \\
\hline SAT CT density (HU) & -96.34 & -93.66 & - & - \\
\hline VAT $\left(\mathrm{cm}^{2}\right)$ & 201.5 & 214.2 & 192.9 & 211.2 \\
\hline VAT CT density (HU) & -83.75 & -86.23 & - & - \\
\hline \multicolumn{5}{|l|}{ Outlier 3} \\
\hline $\mathrm{SMA}\left(\mathrm{cm}^{2}\right)$ & 157.1 & 169.4 & 150.5 & 143.5 \\
\hline SMA CT density (HU) & 16.75 & 19.15 & - & - \\
\hline $\operatorname{SAT}\left(\mathrm{cm}^{2}\right)$ & 298.2 & 267.4 & 298.4 & 267.5 \\
\hline
\end{tabular}

CT, computed tomography; MRI, magnetic resonance imaging; SMA, skeletal muscle area; HU, Hounsfield units; SAT, subcutaneous adipose tissue; VAT, visceral adipose tissue. 